\title{
Optimized microfiber-based third harmonic generation with adaptive control of phase mismatch
}

\author{
Xiujuan Jiang ${ }^{1, *}$, Dandan Zhang ${ }^{1}$, Timothy LeE $^{2}$, And Gilberto Brambilla ${ }^{2}$ \\ ${ }^{1}$ School of Electro-mechanical Engineering, Guangdong University of Technology, Guangzhou, 510006, China \\ ${ }^{2}$ Optoelectronics Research Center, University of Southampton, Southampton, SO17 1BJ, United Kingdom \\ *Corresponding author: jiangxj@gdut.edu.cn
}

Compiled May 20, 2018

\begin{abstract}
An effective approach is presented to improve intermodal third harmonic generation in microfibers. It is demonstrated that structure-independent incident pump power could be utilized, via its effect on nonlinear phase modulations, to compensate for the phase mismatch caused by diameter deviation. The output harmonic of a fabricated microfiber can be optimized adaptively; thus, efficient third harmonic generation with efficiency reaching several percent could realistically be achieved. (๑) 2018 Optical Society of America
\end{abstract}

OCIS codes: (190.0190) Nonlinear optics;(190.2620) Harmonic generation and mixing; (190.4370) Nonlinear optics, fibers.

http://dx.doi.org/10.1364/ao.XX.XXXXXX

Fiber-based third-harmonic generation (THG) has drawn much attention since its first observation in an elliptical-core optical fiber in 1983 [1]. As an all-fiber system has the attractive prospect of providing a robust and cost-effective solution for frequency conversion, this phenomenon has been investigated in different types of fibers [2-11]. However, due to the very low reported conversion efficiencies to date, there is still a long way to go before this solution could become a realistic alternative to the conventional technologies exploiting nonlinear crystals.

In order to overcome the chromatic dispersion, intermodal phase matching has been proposed as a promising technical scheme for fiber-based THG [3]. In 2005, Grubsky et al. theoretically suggested that such scheme, with an appropriate fiber diameter, could achieve an ideal conversion efficiency higher than $80 \%$ with a silica microfiber a few centimeters long [4]. Yet, the efficiency they achieved in the following experimental verification was much lower than the prediction [12]. In fact, despite efforts such as using longer microfiber and resonant enhancement, efficiencies reported in different experiments based on this idea are currently on the order of $10^{-6}-10^{-3}$ [13-15].

Studies have shown that the THG process is critically sensitive to the diameter deviation, and thus intrinsic surface roughness from the microfiber fabrication seriously reduces the conversion efficiency [16]. Microfibers cannot be consistently and repeatably tapered with high precision either. Therefore, efficient THG would actually be impossible to achieve if the phase matching involves solely the microfiber diameter control. In this
Letter, a model of diameter variations more realistic than that in Ref. [16] is presented; we demonstrate that other parameters can be introduced via their effect on nonlinear phase modulations, and they could be utilized to adaptively compensate for the random phase mismatch caused by surface roughness, thus enhancing the THG conversion efficiency.

The THG process in a microfiber can be modeled by the following coupled-mode equations [4]

$$
\begin{aligned}
\partial A_{1} / \partial z= & \text { in }^{(2)} k_{1}\left[\left(J_{1}\left|A_{1}\right|^{2}+2 J_{2}\left|A_{3}\right|^{2}\right) A_{1}+J_{3} A_{1}^{* 2} A_{3} \exp (i \delta \beta z)\right] \\
& -\left(\alpha_{1} / 2\right) A_{1}
\end{aligned}
$$

$$
\begin{aligned}
\partial A_{3} / \partial z= & i n^{(2)} k_{1}\left[\left(6 J_{2}\left|A_{1}\right|^{2}+3 J_{5}\left|A_{3}\right|^{2}\right) A_{3}+J_{3}^{*} A_{1}^{3} \exp (-i \delta \beta z)\right] \\
& -\left(\alpha_{3} / 2\right) A_{3}
\end{aligned}
$$

where the subscript $j=1,3$ refers to the pump/third harmonic; $A_{j}$ is the amplitude of the mode field normalized to its power, i.e., $\left|A_{j}\right|^{2}=P_{j} ; n^{(2)}$ is the nonlinear refractive index coefficient; $k_{1}=2 \pi / \lambda_{1}$ is the pump propagation constant in vacuum; $J_{m}$ are nonlinear overlap integrals (given in detail in Ref. [4]), of which $J_{3}$ gives the overlap between the pump and the harmonic modes, $J_{1}$ and $J_{5}$ govern self-phase modulation (SPM) of the pump and the harmonic respectively, whilst $J_{2}$ relates to crossphase modulation (XPM); $\alpha_{j}$ is the power loss in the microfiber. The propagation constant of the mode in the fiber is defined as $\beta_{j}=\left(\omega_{j} / c\right) n_{j}^{e f f}$, where $\omega_{j}$ is angular frequency, $c$ speed of light in vacuum, and $n_{j}^{\text {eff }}$ the effective refractive index of the mode; then $\delta \beta=\beta_{3}-3 \beta_{1}$ is the propagation constant mismatch between the pump and the third harmonic in fiber. In this Letter, a silica microfiber with $n^{(2)}=2.7 \times 10^{-20} \mathrm{~m}^{2} / \mathrm{W}$ is used, and the pump is a quasi-continuous wave with $\lambda_{1}=1550 \mathrm{~nm}$, which is justified for a ns pump pulse duration.

Fig. 1(a) shows the dependence of $n^{\text {eff }}$ of several modes on the microfiber diameter. We limit our discussion to THG between modes $\operatorname{HE}_{11}\left(\omega_{1}\right)$ and $\operatorname{HE}_{12}\left(3 \omega_{1}\right)$. As they share the same $n^{\text {eff }}$ at $d_{0}=766.48 \mathrm{~nm}, \delta \beta\left(d_{0}\right)=0$. If SPM $/ \mathrm{XPM}$ were ignored now, the third harmonic would grow monotonically with the propagation distance, and most of the pump energy would ideally be converted into the harmonic at $\eta \sim 1$ [see Curve 1 in Fig. 1(b)]; however, the detuning caused by SPM/XPM actually plays 

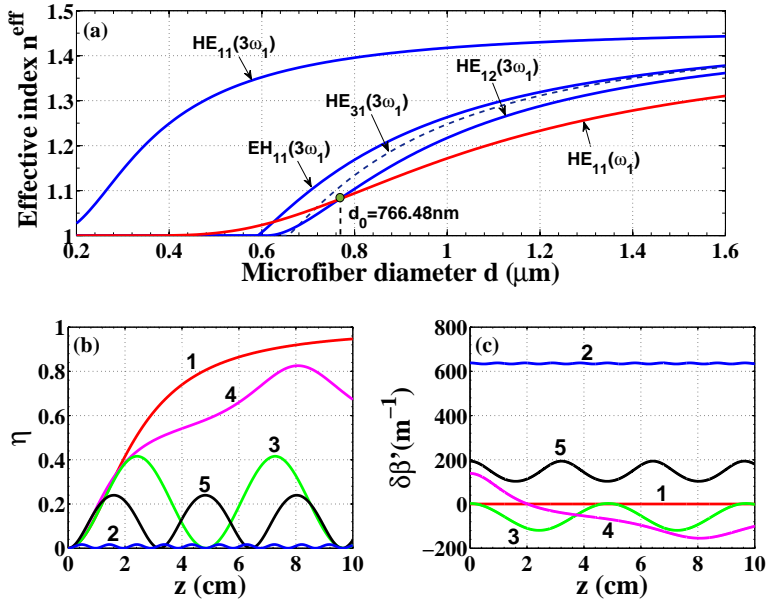

Fig. 1. (a) Dependence of effective refractive index on microfiber diameter for a 1550nm pump wavelength. (b) Conversion efficiency of THG between $\mathrm{HE}_{11}\left(\omega_{1}\right)$ and $\mathrm{HE}_{12}\left(3 \omega_{1}\right)$ and (c) corresponding total propagation constant mismatch against the propagation distance (parameters for different curves are presented in Tables 1 and 2).

an important role, and when these nonlinear phase shifts are included, the total phase-matching condition is not satisfied with $\delta \beta=0$, resulting in a rather poor THG performance (see Curve 2 ). Here, the conversion efficiency is defined as $\eta=P_{3} / P_{0}$, and $P_{0}=\left|A_{1}(0)\right|^{2}$ is the peak incident pump power.

Table 1. Parameters for Curves in Figs. 1(b) and 1(c)

\begin{tabular}{cccccc}
\hline Curve & $d(\mathrm{~nm})$ & $P_{0}(\mathrm{~W})$ & $\delta \beta\left(\mathrm{m}^{-1}\right)$ & SPM/XPM & Loss \\
\hline 1 & 766.48 & 1000 & 0 & ignored & no \\
2 & 766.48 & 1000 & 0 & included & no \\
3 & 766.30 & 1000 & -635 & included & no \\
4 & 766.30 & 1213 & -635 & included & no \\
5 & 766.30 & 1300 & -635 & included & no \\
\hline
\end{tabular}

Table 2. Integrals $J$ for Curves in Figs. 1(b) and 1(c)

\begin{tabular}{cccccc}
\hline \multirow{2}{*}{ Curve } & \multirow{2}{*}{$d(\mathrm{~nm})$} & \multicolumn{5}{c}{$J\left(\mu \mathrm{m}^{-2}\right)$} \\
\cline { 3 - 6 } & & $J_{1}$ & $J_{2}$ & $J_{3}=J_{3}^{*}$ & $J_{5}$ \\
\hline 1 & 766.48 & 0 & 0 & 0.3850 & 0 \\
2 & 766.48 & 0.9742 & 1.4589 & 0.3850 & 3.9682 \\
$3 / 4 / 5$ & 766.30 & 0.9734 & 1.4578 & 0.3847 & 3.9660
\end{tabular}

The total propagation constant mismatch is $\delta \beta^{\prime}=\delta \beta+$ $\delta \beta_{N L}$, where $\delta \beta_{N L}$ is the implicit nonlinear detuning caused by SPM/XPM which can be expressed as

$$
\delta \beta_{N L}(z)=3 k_{1} n^{(2)}\left[\left(2 J_{2}-J_{1}\right) P_{1}(z)+\left(J_{5}-2 J_{2}\right) P_{3}(z)\right],
$$

and it is dominated by two parameters: 1) the fiber diameter $d$ via $J_{1}, J_{2}$ and $J_{5} ; 2$ ) the incident pump power $P_{0}$ via $P_{1}$ and $P_{3}$ (particularly, $P_{1}+P_{3}=P_{0}$ for a lossless fiber). $\delta \beta_{N L}(z)$ is not easy to be dealt with rigorously because it depends on the pump and harmonic powers which vary with propagation distance. An approximation can be made under the condition $\left|A_{3}\right|<<\left|A_{1}\right|$ and by ignoring the fiber loss:

$$
\delta \beta_{N L 0}=3 k_{1} n^{(2)}\left(2 J_{2}-J_{1}\right) P_{0}
$$

and thus a modification of diameter can be made at the beginning to compensate for $\delta \beta_{N L O}$ [4]. The procedure requires first estimating the nonlinear detuning as $\delta \beta_{N L 0}\left(d_{0}, P_{0}\right)$, and then adjusting the diameter to $d_{0}^{\prime}$, so that $\delta \beta\left(d_{0}^{\prime}\right)=-\delta \beta_{N L 0}\left(d_{0}, P_{0}\right)$. With the reduced total mismatch $\delta \beta^{\prime}\left(d_{0}^{\prime}, P_{0}\right)=-\delta \beta_{N L 0}\left(d_{0}, P_{0}\right)+$ $\delta \beta_{N L}\left(d_{0}^{\prime}, P_{0}\right)$, better THG performance can be achieved. In Fig. 1(b), we estimate the detuning with $P_{0}=1000 \mathrm{~W}$ and adjust the diameter to $d_{0}^{\prime}=766.30 \mathrm{~nm}$, then the THG is enhanced as expected (see Curve 3). As $\delta \beta_{N L}$ is diameter dependent, the real detuning experienced in the THG process will have deviated from the estimation after the adjustment; improvement could be possible by conducting an iterative procedure to optimize the diameter so that $\delta \beta^{\prime}\left(d_{\mathrm{opt}}, P_{0}\right) \approx 0$.

This approach for SPM/XPM correction has been employed in almost all the microfiber-based THG work [4, 12-15], but the realistic $\eta$ are much lower than expected. Two reasons may account for this fact. First, the model given by Eq. 3 could not describe the real nonlinear detuning well enough, as the condition $\left|A_{3}\right|<<\left|A_{1}\right|$ would not be satisfied with the harmonic increasing; ignoring the fiber loss would also introduce an error. Second, even if the optimal diameter $d_{\text {opt }}$ could be designed precisely, it would be practically unachievable due to microfiber roughness.

Fortunately, Eq. 3 shows that the nonlinear detuning can also be adjusted by modifying the incident pump power $P_{0}$. This is true even for the original model given by Eq. 2, because the pump power $P_{1}(z)$ and harmonic power $P_{3}(z)$ relate to $P_{0}$. An easy and direct control can be conducted on $P_{0}$ since it is independent of the microfiber structure: in Fig. 1(b), the THG of Curve 3 can be improved with $P_{0}=1213 \mathrm{~W}$ (see Curve 4); however, the performance deteriorates when $P_{0}$ is further raised to 1300W (see Curve 5). Fig. 1(c) presents the real total propagation constant mismatch $\delta \beta^{\prime}(z)$ experienced during the THG process in the above five cases.

Figures 1(b) and 1(c) indicate two important facts. First, once the harmonic power begins to oscillate due to phase mismatch, it does not grow continuously along the propagation direction, so one cannot always expect greater THG output by using a longer microfiber. Second, when the incident pump power results in large total phase mismatch via SPM/XPM, the harmonic power oscillates, so one cannot always expect greater THG output by raising the pump power either. Yet, the harmonic performance could be improved by jointly controlling the microfiber diameter, the microfiber length, and the incident pump power to change the total phase detuning.

The THG performance can be evaluated from two characteristic features: the coherence length $L_{c}=\pi / \delta \beta^{\prime}$ over which the harmonic can grow and the maximum conversion efficiency $\eta_{\max }$. Smaller phase mismatches will generate longer $L_{\mathcal{c}}$ and hence higher $\eta_{\max }$. Figure 2(a) shows the $\eta_{\max }$ within a $10-\mathrm{cm}-$ long microfiber with $d_{0}^{\prime}=766.30 \mathrm{~nm}$ at different $P_{0}$. A higher $\eta_{\max }$ can be achieved by raising $P_{0}$ in a wide range; but when the real nonlinear detuning is significant, the appreciable phase mismatch results in a shorter $L_{c}$, and thus $\eta_{\max }$ declines. The best THG performance appears at $P_{0}=1213 \mathrm{~W}$, with the harmonic increasing along an $8 \mathrm{~cm}$ distance and generating an efficiency 
of about $83 \%$. Figure 2(b) shows the dependence of $\eta$ on $P_{0}$ and the interaction length along the microfiber.
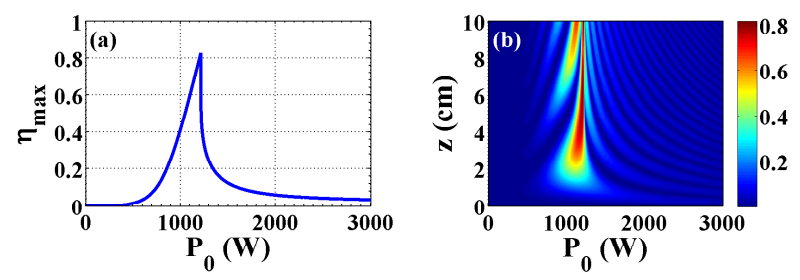

Fig. 2. THG behavior for uniform diameter microfiber. (a) Maximum conversion efficiency within a $10 \mathrm{~cm}$-long microfiber against the incident pump power $P_{0}$. (b) Conversion efficiency against $P_{0}$ (horizontal) and the propagation distance (vertical). The microfiber diameter is $766.30 \mathrm{~nm}$, and loss is ignored.

In Figs. 1(b), 1(c) and 2, the microfiber is ideally uniform along its length. Since intrinsic roughness at the silica microfiber surface is unavoidable, diameter deviations should be considered when exploring the realistic effect of the incident pump power. Figure 3(a) shows a typical random deviation from the median microfiber diameter, where the amplitude $\varepsilon$ is assumed to obey the Gaussian distribution on long distances, i.e.,

$$
p_{1}(\varepsilon)=\frac{1}{\sigma \sqrt{2 \pi}} \exp \left(-\frac{\varepsilon^{2}}{2 \sigma^{2}}\right)
$$

in which $p_{1}$ is the distribution density, and $\sigma$ is the width of the distribution [see Fig. 3(b)]. The existing work has suggested a roughness of about $0.3 \mathrm{~nm}$ [16], and in this Letter, the THG performance will be studied with microfibers of different roughness: (I) $\sigma=0.15 \mathrm{~nm}$, (II) $\sigma=0.3 \mathrm{~nm}$, and (III) $\sigma=0.6 \mathrm{~nm}$. Figure 3(c) shows the linear relation between $\delta \beta$ and the diameter deviation from $d_{0}^{\prime}=766.30 \mathrm{~nm}$.

$\varepsilon$ is assumed to remain constant in a short segment $\Lambda$ along the fiber. This is termed the roughness length and also varies according to a Gaussian probability distribution given by

$$
p_{2}(\Lambda)=\frac{1}{w \sqrt{2 \pi}} \exp \left[-\frac{\left(\Lambda-\Lambda_{0}\right)^{2}}{2 w^{2}}\right]
$$

where $\Lambda_{0}$ is the central roughness length, and $w$ is the width of the distribution ( $w=\Lambda_{0} / 10$ in the simulation). As studies indicate that losses in silica microfibers at $1550 \mathrm{~nm}$ are on the order of $10^{-3}-10^{-2} \mathrm{~dB} / \mathrm{mm}[17,18]$, here we set $\alpha_{1}=0.46 / \mathrm{m}$ (i.e. $2 \times 10^{-3} \mathrm{~dB} / \mathrm{mm}$ ) for the pump; losses would be higher for the shorter wavelength due to greater material absorption [19] and the higher order mode experiencing greater scattering loss from surface roughness, so a rough value of $\alpha_{3}=4.6 / \mathrm{m}$ (i.e. $2 \times 10^{-2} \mathrm{~dB} / \mathrm{mm}$ ) is used for the third harmonic.

Figure 4 (a) shows the $\eta_{\max }$ within a 5 -cm-long rough microfiber when adjusting $P_{0}$ in the range [0,3000W]. Compared with the ideal case in Fig. 2(a), the $\eta_{\max }$ is reduced by more than one order of magnitude, and the optimum incident pump moves towards the high power end because the diameter deviation gives a $\delta \beta$ which needs larger nonlinear phase to compensate; moreover, a greater roughness tends to result in a lower $\eta_{\max }$. The irregular curve profiles are due to the random roughness, and the peaks indicate that better THG performance could be achieved at some incident pump powers.

To give more details of the THG process in microfibers, Figs. 4(b)-4(d), for instance, show the harmonic evolution against $z$

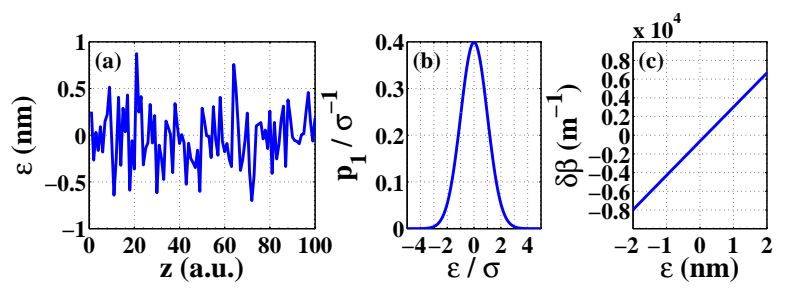

Fig. 3. (a) Diameter deviation of a microfiber with random roughness amplitude $\varepsilon$. (b) Gaussian distribution of $\varepsilon$. (c) Propagation constant mismatch against $\varepsilon$ (from $766.30 \mathrm{~nm}$ ).

at specific $P_{0}$ in the case $\sigma=0.3 \mathrm{~nm}$. With $P_{0}=1000 \mathrm{~W}$, the harmonic winds up first and reaches $\eta_{\max } \sim 6 \times 10^{-3}$ at $z \approx$ $1.7 \mathrm{~cm}$, which accounts for Point A on the corresponding curve in Fig. 4(a), but oscillation on a large scale arises after that and stops the harmonic from increasing further. With $P_{0}=$ $1450 \mathrm{~W}$, i.e. Point B in Fig. 4(a), $\eta_{\max } \sim 5 \times 10^{-3}$ appears at $z \approx$ $0.6 \mathrm{~cm}$, then the harmonic declines along the distance, and the efficiency is as low as $\eta \sim 10^{-5}$ at $z \approx 4.3 \mathrm{~cm}$. With $P_{0}=2352 \mathrm{~W}$, the harmonic basically increases along the whole fiber except for some variation due to the random diameter deviation, and $\eta_{\max } \sim 0.045$ accounts for Point C in Fig. 4(a).
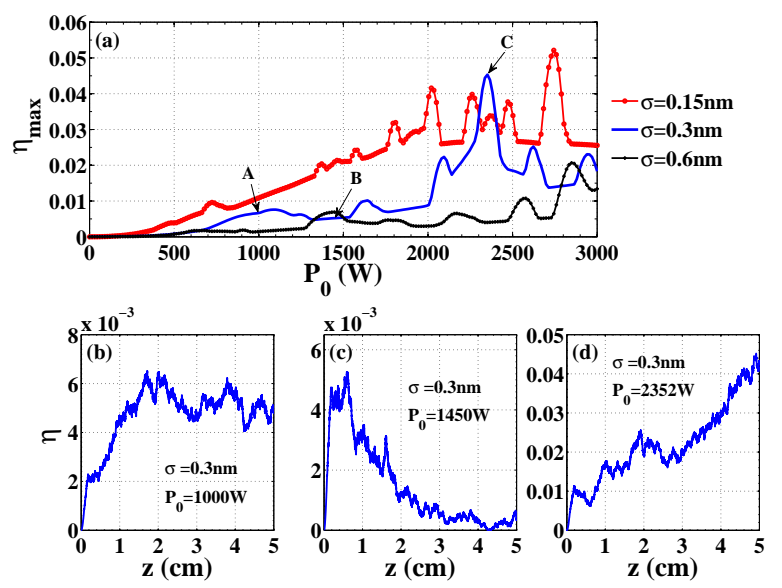

Fig. 4. (a) Maximum conversion efficiency within a 5-cm-long lossy microfiber with different roughness against the incident pump power. The expected diameter is $d_{0}^{\prime}=766.30 \mathrm{~nm}$, and $\Lambda_{0}=10 \mu \mathrm{m}$. (b), (c) and (d) present details of the conversion against the propagation distance at three incident pump powers for $\sigma=0.3 \mathrm{~nm}$.

Since the harmonic power may oscillate along the distance, for a given fiber length it may assume any value between the peak and the minimum, depending on the oscillation period. Hence, $P_{0}$ will influence the output harmonic by altering the phase mismatch to change the oscillation period. Figures 5(a) and 5(b) show its effect on the output harmonic of different microfibers. For lengths $L=1 \mathrm{~mm}, 2 \mathrm{~mm}$, and $5 \mathrm{~mm}$, as the fiber is shorter than $L_{c}$ in the range $[0,3000 \mathrm{~W}]$, the output efficiency increases monotonically with $P_{0}$. For $L=2 \mathrm{~cm}, 5 \mathrm{~cm}$, and $10 \mathrm{~cm}$, with longer interaction length, the overall output could be higher, but the effect of $P_{0}$ becomes complicated: there are some efficient conditions, e.g., with $L=5 \mathrm{~cm}$, the output efficiency reaches $\eta \sim$ 0.043 at $P_{0}=2352 \mathrm{~W}$; but at $1462 \mathrm{~W}$, a low value $\eta \sim 2 \times 10^{-4}$ appears because the output end of the fiber locates near the 
oscillation minimum in the harmonic evolution.

Microfibers with $d(z)=d_{0}^{\prime}+\varepsilon(z)$ generated randomly using identical roughness amplitude and length probability profiles may differ from one another; thus, more simulations have been made to see how much the THG behavior might vary among microfibers. Figures 5(c) and 5(d), respectively, show the output THG for three 5-mm-long and three 5-cm-long microfibers of different random roughness with $\sigma=0.3 \mathrm{~nm}$ and $\Lambda_{0}=10 \mu \mathrm{m}$. Although the efficiency may peak at different $P_{0}$ for each specific microfiber, the trend is similar. Therefore, for a fabricated microfiber with fixed median diameter, roughness and length, by adjusting $P_{0}$ to compensate for the phase detuning adaptively, the THG output can be controlled and optimized.
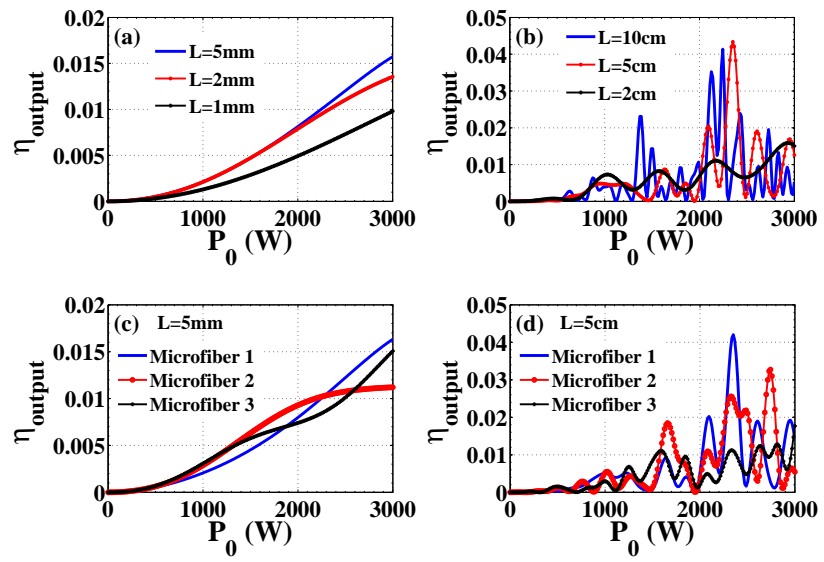

Fig. 5. Output THG conversion efficiency against the incident pump power. (a) Three short and (b) three long microfibers; (c) three 5-mm-long and (d) three 5-cm-long microfibers of different random roughness. The lossy microfibers are $766.30 \mathrm{~nm}$ in diameter, $\sigma=0.3 \mathrm{~nm}, \Lambda_{0}=10 \mu \mathrm{m}$.

So far, the central roughness length has been set to $\Lambda_{0}=$ $10 \mu \mathrm{m}$. Lengths $\Lambda_{0}=50 \mu \mathrm{m}$ and $5 \mu \mathrm{m}$ have also been studied, and the output efficiencies against $P_{0}$ are compared in Fig. 6. With the same $\Lambda_{0}$, a greater roughness amplitude tends to result in a lower minimum/maximum efficiency, while with certain $\sigma$ but longer $\Lambda_{0}$, a higher efficiency seems likely to be achieved at a lower $P_{0}$, which is reasonable since a roughness with longer length, within which the amplitude $\varepsilon$ is assumed to be constant, is less random and thus could be compensated more easily.

Simulations also show that, while efficiency curves for fibers of random $\varepsilon$ distributions (even with identical $\sigma$ ) may have quite different structures, as in Figs. 5(c) and 5(d), results from just random $\Lambda$ distributions (with the same $\Lambda_{0}$ ) are consistent but trivial difference in detail, so the roughness length has less influence compared to the amplitude. The results in Figs. 4(a), 5(a), 5 (b) and 6 are averaged over 10 realizations of random $\Lambda$ while with fixed $\varepsilon$ distribution. Smaller harmonic losses $\alpha_{3}=0.46 / \mathrm{m}$ and $0.046 / \mathrm{m}$ were also tried, with which, although the peak values are slightly different, the $\eta_{\text {output }}$ curves do not change in structure.

To conclude, efficient microfiber-based THG would be unachievable realistically if the intermodal phase matching involves solely diameter control, and surface roughness may result in random THG output varying by several orders of magnitude. Fortunately, some parameters independent of fiber structure (e.g. incident pump power) can be utilized to compensate for the random mismatch adaptively via nonlinear phase shifts caused by
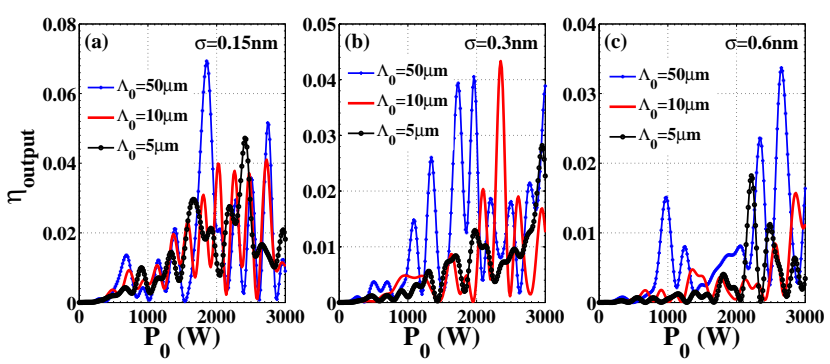

Fig. 6. Output THG conversion efficiency of a 5-cm-long microfiber with different $\sigma$ and $\Lambda_{0}$ against the incident pump power. The lossy microfibers are $766.30 \mathrm{~nm}$ in diameter.

SPM/XPM; thus, the THG output of a fabricated microfiber can be controlled and optimized. A reliable conversion efficiency of a couple of percent (higher than the best experimental value to date by more than ten times) is expected at peak incident pump powers lower than $2000 \mathrm{~W}$ with a silica microfiber several centimeters long. This novel idea provides a simple, effective way to implement THG, and could be applied to other waveguidebased frequency conversion processes.

\section{FUNDING}

National Natural Science Foundation of China (NSFC) (11204043); China Scholarship Council (CSC) (201308440325); Royal Society (IE131732); Engineering and Physical Sciences Research Council (EPSRC) (EP/L01243X/1).

\section{REFERENCES}

1. J. M. Gabriagues, Opt. Lett. 8, 183(1983).

2. D. Nicácio, E. Gouveia, N. Borges, and A. Gouveia-Neto, Appl. Phys. Lett. 62, 2179 (1993).

3. A. Efimov, A. J. Taylor, F. G. Omenetto, J. C. Knight, W. J. Wadsworth, and P. St. J. Russell, Opt. Express 11, 2567 (2003).

4. V. Grubsky and A. Savchenko, Opt. Express 13, 6798 (2005).

5. J. Nold, P. Hölzer, N. Y. Joly, G. K. L. Wong, A. Nazarkin, A. Podlipensky, M. Scharrer, and P. St. J. Russell, Opt. Lett. 35, 2922 (2010).

6. A. Lin, A. Ryasnyanskiy, and J. Toulouse, Opt. Lett. 36, 3437 (2011).

7. K. Tarnowski, B. Kibler, C. Finot, and W. Urbanczyk, IEEE J. Quantum Elect. 47, 622 (2011).

8. K. Bencheikh, S. Richard, G. Mélin, G. Krabshuis, F. Gooijer, and J. A. Levenson, Opt. Lett. 37, 289 (2012).

9. T. Cheng, W. Gao, M. Liao, Z. Duan, D. Deng, M. Matsumoto, T. Misumi, T. Suzuki, and Y. Ohishi, Opt. Lett. 39, 1005 (2014).

10. S. Warren-smith, J. Wie, M. Chemnitz, R. Kostecki, H. Ebendorffheidepriem, T. Monro, and M. Schmidt, Opt. Express 24,17860 (2016).

11. A. Cavanna, F. Just, X. Jiang, G. Leuchs, M. V. Chekhova, P. ST. J. Russell, and N. Y. Joly, Optica 3, 952 (2016).

12. V. Grubsky and J. Feinberg, Opt. Commun. 274, 447 (2007).

13. A. Coillet and P. Grelu, Opt. Commun. 285, 3493 (2012).

14. T. Lee, Y. Jung, C. A. Codemard, M. Ding, N. G. R. Broderick, and G. Brambilla, Opt. Express 20, 8503 (2012).

15. R. Ismaeel, T. Lee, M. Ding, N. Broderick, and G. Brambilla, Opt. Lett. 37, 5121 (2012).

16. M. I. M. A. Khudus, T. Lee, P. Horak, and G. Brambilla, Opt. Lett. 40, 1318 (2015).

17. G. Brambilla, J. Optics 12, 043001 (2010).

18. G. Zhai and L. Tong, Opt. Express 15, 13805 (2007).

19. G. P. Agrawal, Nonlinear Fiber Optics (Academic, 2012). 


\section{REFERENCES}

1. J. M. Gabriagues, "Third-harmonic and three-wave sum-frequency light generation in an elliptical-core optical fiber," Opt. Lett. 8(3), 183-185 (1983).

2. D. Nicácio, E. Gouveia, N. Borges, and A. Gouveia-Neto, "Thirdharmonic generation in $\mathrm{GeO}_{2}$-doped silica single-mode optical fibers," Appl. Phys. Lett. 62(18), 2179-2181 (1993).

3. A. Efimov, A. J. Taylor, F. G. Omenetto, J. C. Knight, W. J. Wadsworth, and P. St. J. Russell, "Phase-matched third harmonic generation in microstructured fibers," Opt. Express 11(20), 2567-2576 (2003).

4. V. Grubsky and A. Savchenko, "Glass micro-fibers for efficient third harmonic generation," Opt. Express 13(18), 6798-6806 (2005).

5. J. Nold, P. Hölzer, N. Y. Joly, G. K. L. Wong, A. Nazarkin, A. Podlipensky, M. Scharrer, and P. St. J. Russell, "Pressure-controlled phase matching to third harmonic in Ar-filled hollow-core photonic crystal fiber," Opt. Lett. 35(17), 2922-2924 (2010).

6. A. Lin, A. Ryasnyanskiy, and J. Toulouse, "Tunable third-harmonic generation in a solid-core tellurite glass fiber," Opt. Lett. 36(17), 34373439 (2011).

7. K. Tarnowski, B. Kibler, C. Finot, and W. Urbanczyk, "Quasi-phasematched third harmonic generation in optical fibers using refractiveindex gratings," IEEE J. Quantum Elect. 47(5), 622-629 (2011).

8. K. Bencheikh, S. Richard, G. Mélin, G. Krabshuis, F. Gooijer, and J. A. Levenson, "Phase-matched third-harmonic generation in highly germanium-doped fiber," Opt. Lett. 37(3), 289-291 (2012).

9. T. Cheng, W. Gao, M. Liao, Z. Duan, D. Deng, M. Matsumoto, T. Misumi, T. Suzuki, and Y. Ohishi, "Tunable third-harmonic generation in a chalcogenide-tellurite hybrid optical fiber with high refractive index difference," Opt. Lett. 39(4), 1005-1007 (2014).

10. S. C. Warren-smith, J. Wie, M. Chemnitz, R. Kostecki, H. Ebendorffheidepriem, T. M. Monro, and M. A. Schmidt, "Third harmonic generation in exposed-core microstructured optical fibers," Opt. Express 24(16), 17860-17867 (2016).

11. A. Cavanna, F. Just, X. Jiang, G. Leuchs, M. V. Chekhova, P. ST. J. Russell, and N. Y. Joly, "Hybrid photonic-crystal fiber for singlemode phase matched generation of third harmonic and photon triplets," Optica 3(9), 952-955 (2016).

12. V. Grubsky and J. Feinberg, "Phase-matched third-harmonic UV generation using low-order modes in a glass micro-fiber," Opt. Commun. 274(2), 447-450 (2007).

13. A. Coillet and P. Grelu, "Third-harmonic generation in optical microfibers: From silica experiments to highly nonlinear glass prospects," Opt. Commun. 285(16), 3493-3497 (2012).

14. T. Lee, Y. Jung, C. A. Codemard, M. Ding, N. G. R. Broderick, and G. Brambilla, "Broadband third harmonic generation in tapered silica fibres," Opt. Express 20(8), 8503-8511 (2012).

15. R. Ismaeel, T. Lee, M. Ding, N. Broderick, and G. Brambilla, "Nonlinear microfiber loop resonators for resonantly enhanced third harmonic generation," Opt. Lett. 37(24), 5121-5123 (2012).

16. M. I. M. A. Khudus, T. Lee, P. Horak, and G. Brambilla, "Effect of intrinsic surface roughness on the efficiency of intermodal phase matching in silica optical nanofibers," Opt. Lett. 40(7), 1318-1321 (2015).

17. G. Brambilla, "Optical fibre nanowires and microwires: a review," J. Optics 12(4), 043001 (2010).

18. G. Zhai and L. Tong, "Roughness-induced radiation losses in optical micro or nanofibers," Opt. Express 15(21), 13805-13816 (2007).

19. G. P. Agrawal, Nonlinear Fiber Optics (Academic, 2012), Chap. 1. 